\title{
A new obstruction of quasialternating links
}

\author{
KHALED QAZAQZEH \\ NAFAA CHBILI
}

\begin{abstract}
We prove that the degree of the $Q$-polynomial of any quasialternating link is less than its determinant. Therefore, we obtain a new and simple obstruction criterion for the link to be quasialternating. As an application, we identify some knots of 12 crossings or less and some links of 9 crossings or less that are not quasialternating. Our obstruction criterion applies also to show that there are only finitely many Kanenobu knots that are quasialternating. Moreover, we identify an infinite family of Montesinos links that are not quasialternating.
\end{abstract}

$57 \mathrm{M} 27$

\section{Introduction}

The notion of quasialternating links was first introduced by Ozsváth and Szabó in [20]. This class of links appeared in the context of link homology as a natural generalization of alternating links. The set of quasialternating links is defined as follows:

Definition 1.1 The set of quasialternating links $\mathcal{Q}$ is the smallest set satisfying the following properties.

(1) The unknot belongs to $\mathcal{Q}$.

(2) If $L$ is a link with a diagram $D$ containing a crossing $c$ such that

(a) both smoothings of the diagram $D$ at the crossing $c, L_{0}$ and $L_{\infty}$ as given in Figure 1 belong to $\mathcal{Q}$,

(b) $\operatorname{det}\left(L_{0}\right), \operatorname{det}\left(L_{\infty}\right) \geq 1$,

(c) $\operatorname{det}(L)=\operatorname{det}\left(L_{0}\right)+\operatorname{det}\left(L_{\infty}\right)$,

then $L$ is in $\mathcal{Q}$ and in this case we say that $L$ is quasialternating at the crossing $c$ with quasialternating diagram $D$.

In general, it is not easy to decide whether a link is quasialternating or not by the use of the definition only. A different approach to address this question is to study the 


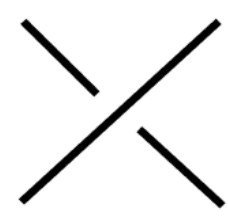

$L$

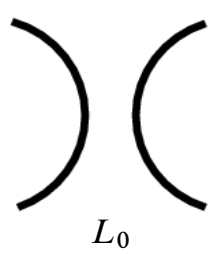

$L_{0}$

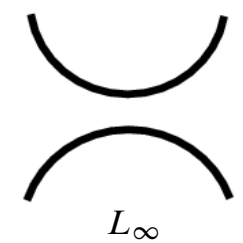

$L_{\infty}$

Figure 1: The diagram of the link $L$ at the crossing $c$ and its smoothings $L_{0}$ and $L_{\infty}$ respectively

behavior of the invariants of quasialternating links in order to find obstruction criteria for a link to be quasialternating. This is actually the main motivation of this paper.

We list below the main obstruction criteria for a link to be quasialternating and the well-known properties that quasialternating links share with alternating links.

(1) The branched double-cover of any quasialternating link is an $L-$-space [20].

(2) The space of branched double-cover of any quasialternating link bounds a negative definite 4-manifold $W$ with $H_{1}(W)=0$ [20].

(3) The $\mathbb{Z} / 2 \mathbb{Z}$ knot Floer homology group of any quasialternating link is thin [17].

(4) The reduced ordinary Khovanov homology group of any quasialternating link is thin [17].

(5) The reduced odd Khovanov homology group of any quasialternating link is thin [19].

In this paper, a new obstruction for a link to be quasialternating is introduced and it is used to provide a table of knots with 12 crossings or less, and a table of links of 9 crossings or less that are not quasialternating. Also, it is used to show that there are only finitely many Kanenobu knots that are quasialternating. This gives an easier proof of one of the claims of Greene and Watson [10, Theorem 2] and supports [8, Conjecture 3.1] of Greene that states that there are only finitely many quasialternating links with a given determinant. Moreover, it is applied to identify an infinite family of Montesinos links that are not quasialternating. This family was conjectured by the authors and Qublan in [22] to be not quasialternating.

\section{Main theorem and its proof}

In 1984, Jones introduced a new Laurent polynomial $V_{L}(t)$ which is an invariant of ambient isotopy of oriented links in the three-sphere. The Jones polynomial can be defined recursively by the following relations: 
(1) If $U$ is the unknot, then $V_{U}(t)=1$.

(2) If $L_{+}, L_{-}$and $L_{0}$ are three links which are identical except in a small ball where they are as given in Figure 2, then

$$
t V_{L_{+}}(t)-t^{-1} V_{L_{-}}(t)=\left(\sqrt{t}+\frac{1}{\sqrt{t}}\right) V_{L_{0}}(t) .
$$
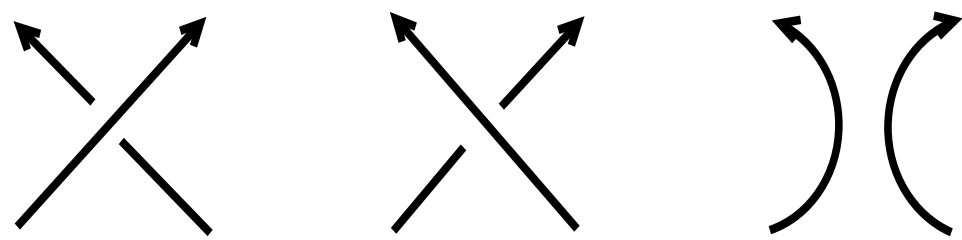

Figure 2: The diagrams of the links $L_{+}, L_{-}$and $L_{0}$, in the small ball respectively

Shortly after the discovery of the Jones polynomial, a new Laurent polynomial $Q_{L}(x)$ is introduced by Brandt, Lickorish and Millett [4] and Ho [11] that is known as the $Q$-polynomial. This polynomial is an invariant of ambient isotopy of unoriented links in the three-sphere that is defined recursively by the following relations:

(1) If $U$ is the unknot, then $Q_{U}(x)=1$.

(2) If $L_{+}, L_{-}, L_{0}$ and $L_{\infty}$ are four link diagrams that are identical except in a small ball where they are as given in Figure 3, then

$$
Q_{L_{+}}(x)+Q_{L_{-}}(x)=x\left(Q_{L_{0}}(x)+Q_{L_{\infty}}(x)\right) .
$$
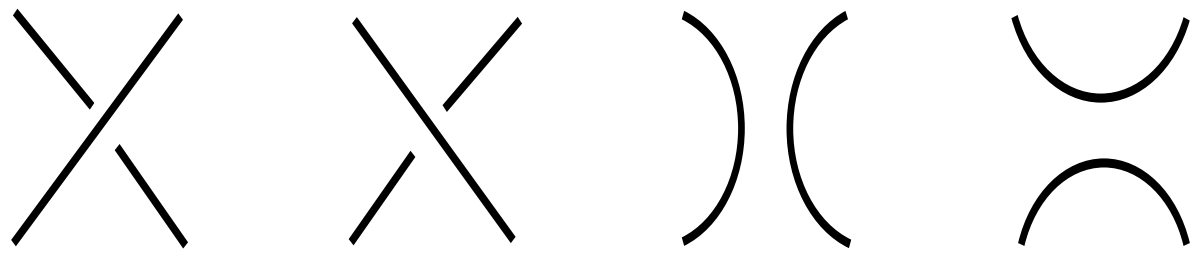

Figure 3: The links $L_{+}, L_{-}, L_{0}$ and $L_{\infty}$, respectively

We point out that the $Q$-polynomial is a specialization of the well known two-variable Kauffman polynomial $F$ that was defined later by Kauffman in [14]. More precisely, for any link $L$, we have $Q_{L}(x)=F_{L}(1, x)$. The degree of the $Q$-polynomial denoted by $\operatorname{deg}(Q)$ is the highest power of this Laurent polynomial. Here are some properties of the $Q$-polynomial that are used in this paper and can be derived from the work in [4]. 
Proposition 2.1 The $Q$-polynomial satisfies the following:

(1) If $L$ is the unlink of $k$-components, then $Q_{L}(x)=\left(2 x^{-1}-1\right)^{k-1}$.

(2) $\operatorname{deg}(Q) \geq 0$.

(3) $Q\left(L^{\prime}\right)=Q(L)$, where $L^{\prime}$ is the mirror image of $L$.

(4) $Q_{L}(2)=(\operatorname{det}(L))^{2}$, where $\operatorname{det}(L)$ is the determinant of the link $L$ that is originally defined from the Seifert matrix and that can be obtained easily from the Alexander and the Jones polynomials of the link.

The following lemma is the key step towards the proof of the main result of this paper.

Lemma 2.1 Let $L$ be a link. Then

$$
\operatorname{deg} Q_{L} \leq \max \left\{\operatorname{deg} Q_{L_{0}}, \operatorname{deg} Q_{L_{\infty}}\right\}+1,
$$

where $L_{0}, L_{\infty}$ are the smoothings of the link $L$ at any crossing $c$.

Proof For any link diagram $D$, we define $n(D)$ to be the length of the switching sequence defined in [14, Section VI] that is the number of crossing switches

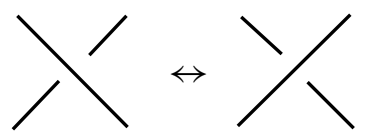

necessary to transform $D$ into a diagram of the unlink. We prove the lemma by induction on $n=n(D)$. Without loss of generality, we can assume that $D=D_{+}$. If $n=1$, then there is a crossing in $D$ whose change produces a diagram $D_{-}$of the unlink with $k$ components $L_{-}$. Since $Q_{L_{-}}(x)=\left(2 x^{-1}-1\right)^{k-1}$, then the skein relation writes $Q_{L}(x)=x\left(Q_{L_{0}}(x)+Q_{L_{\infty}}(x)\right)-\left(2 x^{-1}-1\right)^{k-1}$. Using the fact that the degree of $Q_{L}$ is always nonnegative, we can see that $\operatorname{deg} Q_{L} \leq \max \left\{\operatorname{deg} Q_{L_{0}}, \operatorname{deg} Q_{L_{\infty}}\right\}+1$. Now, let $L$ be a link having a diagram $D$ with a sequence of crossing switches of length $n(D)=n$. Without loss of generality we can assume that $D=D_{+}$and the crossing that we want to apply the skein relation at is the first element of the switching sequence. Therefore, $D_{-}$has a switching sequence of length $n-1$ induced by the switching sequence of $D_{+}$. Now assume that the result is true for all link diagrams with a switching sequence of length less than $n$, in particular for the link diagram $D_{-}$. Again the skein relation writes $Q_{L}(x)=x\left(Q_{L_{0}}(x)+Q_{L_{\infty}}(x)\right)-Q_{L_{-}}(x)$ with $\operatorname{deg} Q_{L}(x) \leq \max \left\{\operatorname{deg}\left(Q_{L_{0}}+Q_{L_{\infty}}\right)+1, \operatorname{deg} Q_{L_{-}}\right\} \leq \max \left\{\operatorname{deg} Q_{L_{0}}, \operatorname{deg} Q_{L_{\infty}}\right\}+1$, where the last equality follows from the induction hypothesis on the link $L_{-}$.

Now we state our main theorem of this paper that gives an obstruction for a link to be quasialternating.

Theorem 2.2 For any quasialternating link $L$, we have $\operatorname{deg} Q_{L}<\operatorname{det}(L)$. 
Proof We use induction on the determinant of the given quasialternating link $L$. The result is obvious if $\operatorname{det}(L)=1$ since the only link that is quasialternating with determinant 1 is the unknot. Now assume that the result is true for all quasialternating links with determinant less than or equal to $m$. If $L$ is a quasialternating link with determinant $m+1$, then both $\operatorname{det}\left(L_{0}\right)$ and $\operatorname{det}\left(L_{\infty}\right)$ are less than or equal to $m$. By the induction assumption $\operatorname{deg} Q_{L_{0}}<\operatorname{det}\left(L_{0}\right)$ and $\operatorname{deg} Q_{L_{\infty}}<\operatorname{det}\left(L_{\infty}\right)$. Consequently,

$\operatorname{deg} Q_{L} \leq \max \left\{\operatorname{deg} Q_{L_{0}}, \operatorname{deg} Q_{L_{\infty}}\right\}+1<\max \left\{\operatorname{det}\left(L_{0}\right), \operatorname{det} L_{\infty}\right\}+1$

$$
<\operatorname{det}\left(L_{0}\right)+\operatorname{det}\left(L_{\infty}\right)=\operatorname{det}(L) \text {. }
$$

\section{Applications and further results}

In [13], Kanenobu introduced an infinite family of knots $K(p, q)$, where $p, q$ are two integers. All these knots are known to have determinant equal to 25 according to the main result of [13]. We want to discuss for which values of $p$ and $q$ the Kanenobu knot $K(p, q)$ is quasialternating. The answer is obtained by applying the obstruction in Theorem 2.2, but first we have to find the degree of the $Q$-polynomial of the Kanenobu knot $K(p, q)$. For this purpose, we need the following proposition.

Proposition 3.1 [13, Proposition 4.5] Let $Q(a, b)$ be the $Q$-polynomial of the Kanenobu knot $K(a, b)$. Then we have

$$
Q(a, b)=-\sigma_{a} \sigma_{b}\left(Q\left(8_{9}\right)-1\right)+x^{-1}\left(\sigma_{a+1} \sigma_{b+1}+\sigma_{a-1} \sigma_{b-1}\right)\left(Q\left(8_{8}\right)-1\right)+1,
$$

where $Q\left(8_{8}\right)=1+4 x+6 x^{2}-10 x^{3}-14 x^{4}+4 x^{5}+8 x^{6}+2 x^{7}$ and $Q\left(8_{9}\right)=$ $-7+4 x+16 x^{2}-10 x^{3}-16 x^{4}+4 x^{5}+8 x^{6}+2 x^{7}$.

In the proposition above, $\sigma_{n}$ is defined as

$$
\sigma_{n}= \begin{cases}\frac{\alpha^{n}-\beta^{n}}{\alpha-\beta} & \text { if } n>0, \\ 0 & \text { if } n=0 \\ -\frac{\alpha^{-n}-\beta^{-n}}{\alpha-\beta} & \text { if } n<0,\end{cases}
$$

where $\alpha+\beta=x$ and $\alpha \beta=1$.

Now the degree of the $Q$-polynomial of the Kanenobu knot $K(p, q)$ can be derived from the above and it is given in the following proposition that appeared first in [23] by Mansour and the second author and whose proof can be found there:

Proposition 3.2 For the Kanenobu knot $K(p, q)$, we have

$$
\operatorname{deg} Q(p, q)= \begin{cases}|p|+|q|+6 & \text { if } p q \geq 0 \\ |p|+|q|+5 & \text { otherwise. }\end{cases}
$$


The answer to the above question is given in the following corollary.

Corollary 3.3 There are only finitely many Kanenobu knots that are quasialternating.

Proof According to the obstruction in Theorem 2.2, a necessary condition for the Kanenobu knot to be quasialternating, is

$$
\operatorname{deg} Q_{K(p, q)} \leq|p|+|q|+6<25 .
$$

This implies that $|p|+|q|<19$ and we know that there are only finitely many values of $p$ and $q$ that satisfy this inequality.

The following corollary identifies an infinite family of Montesinos links that are not quasialternating.

Corollary 3.4 The Montesinos link $L=M\left(e ;\left(\alpha_{1}, \beta_{1}\right),\left(\alpha_{2}, \beta_{2}\right), \ldots,\left(\alpha_{r}, \beta_{r}\right),(\alpha, \beta)\right)$ for all $\alpha=l+k \beta$ with $k$ large enough and $l=0,1, \ldots, \beta-1$ in standard form is not quasialternating if $e=1$ and $\sum_{i=1}^{r} \beta_{i} / \alpha_{i}=1$.

Proof According to the obstruction in Theorem 2.2, a necessary condition for the above Montesinos link to be quasialternating is

$\operatorname{deg} Q_{L}=c(D)-2=c(L)-2<\operatorname{det}(L)=\left(\alpha \prod_{i=1}^{r} \alpha_{i}\right)\left(-1+\sum_{i=1}^{r} \frac{\beta_{i}}{\alpha_{i}}+\frac{\beta}{\alpha}\right)=\beta \prod_{i=1}^{r} \alpha_{i} ;$

the first two equalities follow from Lickorish and Thistlethwaite [15, Lemma 8, Theorem 10] where $c(L)$ and $c(D)$ are the crossing numbers of the Montesinos link and its corresponding Montesinos reduced link diagram respectively. The third equality follows from the formula to compute the determinant of Montesinos link that first appeared in Champanerkar and Ording [7, Proposition 3.1] and it can be derived from the work in [22]. Note that increasing the value of $k$ will increase the value of $c(D)$ while the determinant stays fixed. Therefore, we can choose $k$ large enough for fixed $\beta$ so that $\beta \prod_{i=1}^{r} \alpha_{i} \leq c(L)-2$.

Corollary 3.5 The above corollary gives a partial solution of [22, Conjecture 3.10].

Proof To show the claim, we have to show that $\alpha_{i} /\left(\alpha_{i}-\beta_{i}\right) \leq \min \left\{\min \left\{\alpha_{j} / \beta_{j} \mid\right.\right.$ $j \neq i\}, \alpha / \beta\}$ for any $1 \leq i \leq r$ and $\alpha /(\alpha-\beta) \leq \min \left\{\alpha_{j} / \beta_{j} \mid 1 \leq j \leq r\right\}$. If we choose $k$ large enough such that $\alpha / \beta=(l / \beta)+k>\alpha_{i} / \beta_{i}$ for all $1 \leq i \leq r$, then it is enough to show that $\alpha_{i} /\left(\alpha_{i}-\beta_{i}\right) \leq \min \left\{\alpha_{j} / \beta_{j} \mid j \neq i\right\}$ for any $1 \leq i \leq r$ and $\alpha /(\alpha-\beta) \leq \min \left\{\alpha_{j} / \beta_{j} \mid 1 \leq j \leq r\right\}$. For the first part, suppose $\alpha_{i} /\left(\alpha_{i}-\beta_{i}\right)>\alpha_{j} / \beta_{j}$ for 
some $j$, then we have $\alpha_{i} \beta_{j}+\alpha_{j} \beta_{i}>\alpha_{j} \alpha_{i}$. This implies that $\beta_{j} / \alpha_{j}+\beta_{i} / \alpha_{i}>1$ which contradicts the assumption. Similarly for the second part suppose that $\alpha /(\alpha-\beta)>$ $\alpha_{m} / \beta_{m}=\min \left\{\alpha_{j} / \beta_{j} \mid 1 \leq j \leq r\right\}$ for some $m$, then we have $(\alpha-\beta) / \alpha<\beta_{m} / \alpha_{m}$. Therefore, we obtain

$$
1-\frac{\beta_{m}}{\alpha_{m}}<1-\frac{\beta}{\alpha}<\frac{\beta_{m}}{\alpha_{m}}
$$

So we conclude that $\frac{1}{2}<\beta_{m} / \alpha_{m}$. Thus, $2<\alpha / \beta=(j+k \beta) /(j+(k-1) \beta)$. This implies that $j+(k-2) \beta<0$ which is a contradiction since $0 \leq j, k-2, \beta$ for large $k$.

Remark Corollary 3.4 explains why most but finitely many of the Montesinos links of the form

$$
\begin{aligned}
L(m, n) & =M\left(0 ;\left(m^{2}+1, m\right),(n, 1),\left(m^{2}+1,-m\right)\right) \\
& =M\left(1 ;\left(m^{2}+1, m\right),\left(m^{2}+1, m^{2}+1-m\right),(n, 1)\right)
\end{aligned}
$$

for positive integers $m, n$ and large $n$ for fixed $m$ are not quasialternating. This phenomena was first explained in [8, Theorem 1.3] for the Montesinos knot $L(2,3)$ which is the knot $11 n 50$. Also, Greene in [8, Subsection 3.2] pointed out that the proof of [8, Theorem 1.3] can be generalized easily to all $L(m, n)$ with $n>m$.

In [16], Manolescu showed that all homologically thin in Khovanov homology nonalternating knots of crossing number less than or equal to 9 are quasialternating, except the knot 946 . Among the 42 nonalternating knots of 10 crossings, 32 are homologically thin in Khovanov homology. Baldwin [1], Champanerkar and Kofman [6], Greene [9] and Manolescu [16] showed that all these knots are quasialternating except for the knot $10_{140}$. Shumakovitch in [25] showed that the knots $9_{46}, 10_{140}$ have thick odd Khovanov homology groups, so they are not quasialternating.

The inequality in Theorem 2.2 does not characterize quasialternating links since the knots $9_{46}, 10_{128}$, and $11 n 50$ for instance, satisfy the inequality $\operatorname{deg}\left(Q_{L}\right)<\operatorname{det}(L)$, but they are not quasialternating. Actually, the knot $10_{128}$ is homologically thick in Khovanov homology; see Bar-Natan and Morrison [2]. The knot $11 n 50$ which is the Kanenobu knot $K(3,0)$ does not bound a negative definite 4 -manifold with torsion-free cohomology as has been shown by Greene in [8, Theorem 1.3].

The following two propositions show that the inequality in Theorem 2.2 is far from characterizing quasialternating links even with assuming an extra condition on the link namely of being homologically thin in Khovanov homology. 
Proposition 3.6 There are two infinite families, one of knots and one of links, which are not quasialternating but satisfy the inequality in Theorem 2.2.

Proof The first family is the set of the pretzel knots of the form $P(r+2, r+1,-r)$ and the second family is the set of the pretzel links $P(r+1, r+1,-r)$, where $r>3$ is an odd integer. It has been shown by the second author in [21] that these knots and links are thick in Khovanov homology. Therefore, they are not quasialternating. However, they satisfy the inequality in Theorem 2.2,

$$
\begin{aligned}
& \operatorname{deg} Q_{P(r+2, r+1,-r)}=3 r+1 \leq r^{2}-2=\operatorname{det}(P(r+2, r+1,-r)), \\
& \operatorname{deg} Q_{P(r+1, r+1,-r)}=3 r+2 \leq r^{2}-1=\operatorname{det}(P(r+1, r+1,-r)),
\end{aligned}
$$

where the first equality in each of the two equations above follows from [15, Lemma 8 , Theorem 10].

Proposition 3.7 There is an infinite family of links that are not quasialternating homologically thin in Khovanov homology and satisfy the inequality in Theorem 2.2.

Proof The family is the set of the pretzel links $P(n, n,-n)$ for $n \geq 3$. It has been shown that all these links are homologically thin in Khovanov homology in [21]. However, they are not quasialternating by [8, Theorem 1.4]. It is left to show that all these links satisfy the inequality in Theorem 2.2. We have

$$
\operatorname{deg} Q_{P(n, n,-n)}=3 n-2 \leq n^{2}=\operatorname{det}(P(n, n,-n)),
$$

for $n \geq 3$, where the first equality of above equation follows from [15, Lemma 8 , Theorem 10].

The breadth of the Jones polynomial of an oriented link breadth $V_{L}(t)$ is defined to be the difference between the highest and the lowest degree of $t$ that appear in $V_{L}(t)$. Inspired by the inequality in Theorem 2.2 and computations of the breadth and the determinants of a large family of links, we conjecture the following.

Conjecture 3.8 If $L$ is a quasialternating link, then breadth $V_{L}(t) \leq \operatorname{det}(L)$.

Conjecture 3.8 is weaker than the main conjecture of Jaradat, the second author and Qublan in [24] which states that the crossing number is a lower bound of the determinant for any quasialternating link, ie $c(L) \leq \operatorname{det}(L)$ since we know that for any link the breadth of the Jones polynomial is always less than or equal to the crossing number. The importance of the latter does also come from the fact that it solves a conjecture of Greene in [8] which states that there are only finitely many quasialternating links 
with a given determinant. However, Conjecture 3.8 has the advantage that it involves the breadth of the Jones polynomial which is, in general, easier to compute than the crossing number. Conjecture 3.8 is true for all quasialternating links that have been checked to satisfy the conjecture $c(L) \leq \operatorname{det}(L)$; see [24]. In the Appendix, we prove both conjectures for quasialternating closed 3 -braids.

We now apply the obstruction criterion introduced in Theorem 2.2 to provide a table of knots of 12 crossings or less and a table of links of 9 crossings or less that are not quasialternating. The computations of the $Q$-polynomials and the determinants are done using the Knot Atlas [2] and Knotinfo [5].

Finally, we close this section with the following two questions:

Question 3.9 Can we determine all Kanenobu knots that are quasialternating?

We conjecture that $K(0,0)=4_{1} \# 4_{1}, K(1,0)=8_{8}, K(1,-1)=8_{9}, K(2,-1)=$ $10_{129}, K(2,0)=10_{137}, K(1,1)=10_{155}$ are the only Kanenobu knots that are quasialternating.

Question 3.10 Can we characterize all quasialternating knots with crossing number less than or equal to 11 ?

Table 1 combined with the table in Jablan [12] gives a partial solution for the above question.

Remark We would like to mention that a refinement of Theorem 2.2 was obtained by M Teragaito in [26] in which he gives a sharper inequality.

\section{Appendix: Proof of Conjecture 3.8 for closed 3-braids}

In this section we prove Conjecture 3.8 for quasialternating closed 3-braids. Although a direct proof can be given by computing the breadth of the Jones polynomial of closed 3 -braids. We prefer here to prove that for any quasialternating link $L$ of braid index less than or equal to 3 , we have $c(L) \leq \operatorname{det}(L)$. This will imply that our conjecture is true for this class of links.

For $n \geq 2$, let $B_{n}$ be the braid group on $n$ strings. It is well known that $B_{n}$ is generated by the elementary braids $\sigma_{1}, \sigma_{2}, \ldots, \sigma_{n-1}$ subject to the relations

$$
\begin{aligned}
\sigma_{i} \sigma_{j} & =\sigma_{j} \sigma_{i} & & \text { if }|i-j| \geq 2, \\
\sigma_{i} \sigma_{i+1} \sigma_{i} & =\sigma_{i+1} \sigma_{i} \sigma_{i+1} & & \text { for all } 1 \leq i \leq n-2 .
\end{aligned}
$$


Knot Det. Deg. Knot Det. Deg. $\quad$ Knot Det. Deg.

\begin{tabular}{|c|c|c|c|c|c|c|c|c|}
\hline $8_{19}$ & 3 & 6 & $9_{42}$ & 7 & 7 & $10_{124}$ & 1 & 8 \\
\hline $10_{132}$ & 5 & 8 & $10_{139}$ & 3 & 8 & $10_{145}$ & 3 & 8 \\
\hline $10_{153}$ & 1 & 8 & $10_{161}$ & 5 & 6 & $11 n 9$ & 5 & 9 \\
\hline $11 n 19$ & 5 & 9 & $11 \ln 31$ & 3 & 9 & $11 n 34$ & 1 & 9 \\
\hline $11 \ln 38$ & 3 & 9 & $11 n 42$ & 1 & 9 & $11 n 49$ & 1 & 9 \\
\hline $11 n 57$ & 7 & 9 & $11 n 67$ & 9 & 9 & $11 n 96$ & 7 & 9 \\
\hline $11 n 102$ & 3 & 9 & $11 n 104$ & 3 & 9 & $11 n 111$ & 7 & 9 \\
\hline $11 n 116$ & 1 & 7 & $11 n 135$ & 5 & 7 & $11 n 139$ & 9 & 9 \\
\hline $12 n 0019$ & 1 & 10 & $12 n 0023$ & 9 & 10 & $12 n 0031$ & 9 & 10 \\
\hline $12 n 0051$ & 9 & 10 & $12 n 0056$ & 9 & 9 & $12 n 0057$ & 9 & 9 \\
\hline $12 n 0096$ & 7 & 10 & $12 n 0118$ & 7 & 10 & $12 n 0121$ & 1 & 10 \\
\hline $12 n 0124$ & 7 & 10 & $12 n 0129$ & 7 & 10 & $12 n 0149$ & 5 & 10 \\
\hline $12 n 0175$ & 3 & 10 & $12 n 0200$ & 9 & 10 & $12 n 0210$ & 1 & 10 \\
\hline $12 n 0214$ & 1 & 10 & $12 n 0217$ & 5 & 10 & $12 n 0221$ & 9 & 9 \\
\hline $12 n 0242$ & 1 & 10 & $12 n 0243$ & 5 & 10 & $12 n 0268$ & 9 & 10 \\
\hline $12 n 0273$ & 5 & 10 & $12 n 0292$ & 1 & 10 & $12 n 0293$ & 7 & 10 \\
\hline $12 n 0309$ & 1 & 10 & $12 n 0313$ & 1 & 10 & $12 n 0318$ & 1 & 8 \\
\hline $12 n 0332$ & 9 & 10 & $12 n 0336$ & 5 & 10 & $12 n 0352$ & 7 & 10 \\
\hline $12 n 0370$ & 5 & 10 & $12 n 0386$ & 9 & 10 & $12 n 0402$ & 9 & 10 \\
\hline $12 n 0403$ & 9 & 10 & $12 n 0404$ & 3 & 10 & $12 n 0419$ & 3 & 10 \\
\hline $12 n 0430$ & 1 & 9 & $12 n 0439$ & 3 & 8 & $12 n 0446$ & 7 & 10 \\
\hline $12 n 0473$ & 1 & 10 & $12 n 0475$ & 7 & 10 & $12 n 0488$ & 5 & 10 \\
\hline $12 n 0502$ & 9 & 10 & $12 n 0519$ & 7 & 8 & $12 n 0552$ & 9 & 9 \\
\hline $12 n 0574$ & 9 & 10 & $12 n 0575$ & 3 & 10 & $12 n 0579$ & 9 & 9 \\
\hline $12 n 0582$ & 9 & 10 & $12 n 0591$ & 7 & 8 & $12 n 0605$ & 9 & 10 \\
\hline $12 n 0617$ & 5 & 9 & $12 n 0644$ & 7 & 8 & $12 n 0655$ & 3 & 8 \\
\hline $12 n 0673$ & 5 & 10 & $12 n 0676$ & 9 & 10 & $12 n 0689$ & 7 & 10 \\
\hline $12 n 0725$ & 5 & 10 & $12 n 0749$ & 7 & 8 & $12 n 0812$ & 9 & 9 \\
\hline $12 n 0815$ & 7 & 9 & $12 n 0851$ & 5 & 8 & & & \\
\hline
\end{tabular}

Table 1: Knot table

\begin{tabular}{ccc|ccc|ccc} 
Link & Det. & Deg. & Link & Det. & Deg. & Link & Det. & Deg. \\
\hline$L 8 n 3$ & 4 & 6 & $L 8 n 6$ & 0 & 6 & $L 8 n 8$ & 0 & 5 \\
$L 9 n 4$ & 4 & 7 & $L 9 n 9$ & 4 & 7 & $L 9 n 12$ & 6 & 7 \\
$L 9 n 15$ & 2 & 7 & $L 9 n 18$ & 2 & 7 & $L 9 n 19$ & 0 & 5 \\
$L 9 n 21$ & 4 & 6 & $L 9 n 27$ & 4 & 7 & & &
\end{tabular}

Table 2: Link table 
The two generators $\sigma_{1}$ and $\sigma_{2}$ of the braid group $B_{3}$ are pictured in Figure 4. Recall that every link $L$ in $S^{3}$ can be obtained as the closure of a certain braid $b$. We write $L=\hat{b}$. Closed 3-braids have been classified up to conjugation by Murasugi in the following theorem [18].
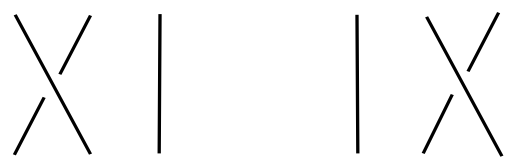

Figure 4: The generators $\sigma_{1}$ and $\sigma_{2}$ of $B_{3}$ respectively

Theorem A.1 Let $b$ be a 3-braid and let $h=\left(\sigma_{1} \sigma_{2}\right)^{3}$ be a full positive twist. Then $b$ is conjugate to exactly one of the following:

(1) $h^{n} \sigma_{1}^{p_{1}} \sigma_{2}^{-q_{1}} \cdots \sigma_{1}^{p_{s}} \sigma_{2}^{-q_{s}}$, where $s, p_{i}$ and $q_{i}$ are positive integers.

(2) $h^{n} \sigma_{2}^{m}$ where $m \in \mathbb{Z}$.

(3) $h^{n} \sigma_{1}^{m} \sigma_{2}^{-1}$, where $m \in\{-1,-2,-3\}$.

Baldwin classified quasialternating closed 3-braids in the following theorem [1]:

Theorem A.2 Let $L$ be a closed 3-braid.

(1) If $L$ is the closure of $h^{n} \sigma_{1}^{p_{1}} \sigma_{2}^{-q_{1}} \cdots \sigma_{1}^{p_{s}} \sigma_{2}^{-q_{s}}$, where $s, p_{i}$ and $q_{i}$ are positive integers, then $L$ is quasialternating if and only if $n \in\{-1,0,1\}$.

(2) If $L$ is the closure of $h^{n} \sigma_{2}^{m}$, then $L$ is quasialternating if and only if either $n=1$ and $m \in\{-1,-2,-3\}$ or $n=-1$ and $m \in\{1,2,3\}$.

(3) If $L$ is the closure of $h^{n} \sigma_{1}^{m} \sigma_{2}^{-1}$ where $m \in\{-1,-2,-3\}$, then $L$ is quasialternating if and only if $n \in\{0,1\}$.

We now introduce explicit formulas for the determinant of any closed 3-braid.

Proposition A.1 (1) Suppose that

$$
L=\overline{h^{n} \sigma_{1}^{p_{1}} \sigma_{2}^{-q_{1}} \cdots \sigma_{1}^{p_{s}} \sigma_{2}^{-q_{s}}},
$$

where $s, p_{i}$ and $q_{i}$ are positive integers. Let $p=\sum_{i=1}^{s} p_{i}$ and $q=\sum_{i=1}^{s} q_{i}$.

(a) If $n$ is odd, then

$$
\begin{aligned}
\operatorname{det}(L)=4+p q+\sum_{\substack{k=2 \\
i_{1}<\cdots<i_{k}}}^{s} p_{i_{1}} \cdots p_{i_{k}}\left(q_{i_{1}}+\cdots+q_{i_{2}-1}\right) \\
\quad \cdots\left(q_{i_{k-1}}+\cdots+q_{i_{k}-1}\right)\left(q-\left(q_{i_{1}}+\cdots+q_{i_{k-1}}\right)\right) .
\end{aligned}
$$


(b) If $n$ is even, then

$$
\begin{aligned}
\operatorname{det}(L)=p q+\sum_{\substack{k=2 \\
i_{1}<\cdots<i_{k}}}^{s} p_{i_{1}} \cdots p_{i_{k}}\left(q_{i_{1}}+\cdots+q_{i_{2}-1}\right) \\
\cdots\left(q_{i_{k-1}}+\cdots+q_{i_{k}-1}\right)\left(q-\left(q_{i_{1}}+\cdots+q_{i_{k-1}}\right)\right) .
\end{aligned}
$$

(2) If $L=\widehat{h^{n} \sigma_{2}^{m}}$ where $m \in \mathbb{Z}$ then $\operatorname{det}(L)=0$ if $n$ is even and $\operatorname{det}(L)=4$ if $n$ is odd.

(3) If $L=\widehat{h^{n} \sigma_{1}^{m} \sigma_{2}^{-1}}$ where $m \in\{-1,-2,-3\}$, then $\operatorname{det}(L)=2$ if $m=-2$ and $\operatorname{det}(L)=2+(-1)^{3 n+m}$ if $m=-1$ or -3 .

Proof In [3], Birman showed that the Jones polynomial of a closed 3-braid $\alpha$ is given by

$$
V_{\widehat{\alpha}}(t)=(-\sqrt{t})^{e_{\alpha}}\left(t+t^{-1}+\operatorname{tr}\left(\psi_{t}(\alpha)\right)\right),
$$

where $e_{\alpha}$ is the exponent sum of $\alpha$ as a word in the elementary braids $\sigma_{1}$ and $\sigma_{2}$. Also, $\psi_{t}: B_{3} \rightarrow \mathrm{GL}\left(2, \mathbb{Z}\left[t, t^{-1}\right]\right)$ is the Burau representation defined by

$$
\psi_{t}\left(\sigma_{1}\right)=\left[\begin{array}{cc}
-t & 1 \\
0 & 1
\end{array}\right] \text { and } \psi_{t}\left(\sigma_{2}\right)=\left[\begin{array}{cc}
1 & 0 \\
t & -t
\end{array}\right]
$$

and tr denotes the usual matrix-trace function. Recall that for any link $L$, we have $\operatorname{det}(L)=\left|V_{L}(-1)\right|$. The values of the determinants in cases (2) and (3) are obtained easily. Indeed, we have explicit formulas for the Jones polynomials in these cases:

$$
\begin{aligned}
V_{h^{n} \sigma_{2}^{m}}(t) & =(-\sqrt{t})^{m+6 n}\left(t+t^{-1}+t^{3 n}+(-1)^{m} t^{3 n+m}\right), \\
V_{h^{n} \sigma_{1}^{-1}} \sigma_{2}^{-1} & (t)=(-\sqrt{t})^{6 n-2}\left(t+t^{-1}+t^{3 n}(-t)^{-1}\right), \\
V_{h^{n} \sigma_{1}^{-2} \sigma_{2}^{-1}}(t) & =(-\sqrt{t})^{6 n-3}\left(t+t^{-1}\right), \\
V_{h^{n} \sigma_{1}^{-3} \sigma_{2}^{-1}}(t) & =(-\sqrt{t})^{6 n-4}\left(t+t^{-1}+t^{3 n}(-t)^{-2}\right) .
\end{aligned}
$$

Let $\beta=\overline{\sigma_{1}^{p_{1}} \sigma_{2}^{-q_{1}} \cdots \sigma_{1}^{p_{s}} \sigma_{2}^{-q_{s}}}$ and $\alpha=h^{n} \beta$. Since $\psi_{t}\left(\sigma_{1} \sigma_{2}\right)^{3}=t^{3} . I_{2}$, then $V_{\widehat{\alpha}}(t)=$ $(-\sqrt{t})^{e_{\alpha}}\left[t+t^{-1}+t^{3 n} \operatorname{tr}\left(\psi_{t}(\beta)\right)\right]$. Consequently, $\operatorname{det}(\widehat{\alpha})=\left|V_{\widehat{\alpha}}(-1)\right|=\mid-1-1+$ $(-1)^{n} \operatorname{tr}\left(\psi_{-1}((\beta)) \mid\right.$. On the other hand, for any positive integers $n$ and $m$, we have

$$
\psi_{-1}\left(\sigma_{1}^{n}\right)=\left[\begin{array}{ll}
1 & n \\
0 & 1
\end{array}\right] \quad \text { and } \quad \psi_{-1}\left(\sigma_{2}^{-m}\right)=\left[\begin{array}{cc}
1 & 0 \\
m & 1
\end{array}\right] .
$$

Hence

$$
\psi_{-1}\left(\sigma_{1}^{n} \sigma_{2}^{-m}\right)=\left[\begin{array}{cc}
1+n m & n \\
m & 1
\end{array}\right] .
$$


A simple induction implies that $\operatorname{tr}\left(\psi_{-1}(\beta)\right)$ is always positive. In conclusion, $\operatorname{det}(\hat{\alpha})=$ $\operatorname{det}(\hat{\beta})$ if $n$ is even and $\operatorname{det}(\hat{\alpha})=\operatorname{det}(\widehat{\beta})+4$, if $n$ is odd.

To prove the formula in the case (1), we first compute the determinant of the alternating link

$$
\widehat{\beta}=\overline{\sigma_{1}^{p_{1}} \sigma_{2}^{-q_{1}} \cdots \sigma_{1}^{p_{s}} \sigma_{2}^{-q_{s}}}
$$

by counting the number of spanning trees of the Tait graph associated with the diagram of $\hat{\beta}$ given in Figure 5. This graph is made up of a cycle $u_{1} u_{2} \cdots u_{q} u_{1}$ together with an extra vertex $w$ of degree $p$ which is connected to every vertex $u_{q_{i}}$ by $p_{i}$ parallel edges.

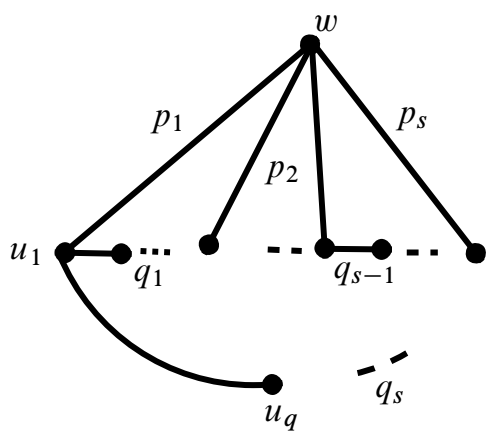

Figure 5: The Tait graph associated with the diagram of $\hat{\beta}$

A spanning tree where $w$ has degree 1 consists of an edge among the $p$ edges incident to $w$ and a spanning tree of the cycle. There are obviously $p q$ such trees. For a spanning tree where $w$ has degree $k \geq 2$, we first make a choice of $k$ mutually nonparallel edges incident to $w$, say $w u_{q_{i_{1}}}, \ldots, w u_{q_{i_{k}}}$. Then we break all the cycles of type $w u_{q_{i_{r}}} \cdots w u_{q_{i_{r+1}}} w$ and the cycle $w u_{q_{i_{k}}} \cdots u_{q_{i_{q}}} w$ by removing from each cycle an edge not incident to $w$. Note that the number of spanning trees will be then the product $p_{i_{1}} \cdots p_{i_{k}}\left(q_{i_{1}}+\cdots+q_{i_{2}-1}\right) \cdots\left(q_{i_{k-1}}+\cdots+q_{i_{k}-1}\right)\left(q-\left(q_{i_{1}} \cdots q_{i_{k}-1}\right)\right)$. The total number of spanning trees is obtained by taking the sum through all $k \leq s$ and $i_{1}<\cdots<i_{k}$. If $n$ is even, then $\operatorname{det}(L)=\operatorname{det}(\hat{\beta})$. However, if $n$ is odd, then $\operatorname{det}(L)=\mid-1-1-\operatorname{tr}\left(\psi_{-1}(\beta) \mid=\operatorname{tr}\left(\psi_{-1}(\beta)\right)+2=\operatorname{det}(\hat{\beta})+4\right.$.

Now, we prove that for all quasialternating closed 3-braids, we have $c(L) \leq \operatorname{det}(L)$. We start by considering the class of links in the first case of Baldwin's theorem. If $n=0$, then the link is alternating and the result holds. We will prove the result for $n=1$, the case $n=-1$ is similar. Assuming that

$$
L=\overline{\left(\sigma_{1} \sigma_{2}\right)^{3} \sigma_{1}^{p_{1}} \sigma_{2}^{-q_{1}} \cdots \sigma_{1}^{p_{s}} \sigma_{2}^{-q_{s}}},
$$


we have the following.

If $s>1$, or $\left(s=1, p_{1}>1\right.$ and $\left.q_{1}>1\right)$, then by the proposition above $\operatorname{det}(L) \geq$ $4+p q \geq 4+p+q$. On the other hand we have

$$
\begin{aligned}
\left(\sigma_{1} \sigma_{2}\right)^{3} \sigma_{1}^{p_{1}} \sigma_{2}^{-q_{1}} \cdots \sigma_{1}^{p_{s}} \sigma_{2}^{-q_{s}} & \equiv\left(\sigma_{2} \sigma_{1}\right)^{3} \sigma_{1}^{p_{1}} \sigma_{2}^{-q_{1}} \cdots \sigma_{1}^{p_{s}} \sigma_{2}^{-q_{s}} \\
& \equiv \sigma_{2} \sigma_{1} \sigma_{2} \sigma_{1} \sigma_{2} \sigma_{1} \sigma_{1}^{p_{1}} \sigma_{2}^{-q_{1}} \cdots \sigma_{1}^{p_{s}} \sigma_{2}^{-q_{s}} \\
& \equiv \sigma_{1} \sigma_{2} \sigma_{1} \sigma_{2} \sigma_{1} \sigma_{1}^{p_{1}} \sigma_{2}^{-q_{1}} \cdots \sigma_{1}^{p_{s}} \sigma_{2}^{-q_{s}+1} .
\end{aligned}
$$

Thus, $c(L) \leq 5+p+q-1=4+p+q \leq \operatorname{det}(L)$.

If $s=1, p=1$ and $q>1$, then $\operatorname{det}(L)=4+q$, and

$$
\begin{aligned}
\left(\sigma_{1} \sigma_{2}\right)^{3} \sigma_{1} \sigma_{2}^{-q} & \equiv \sigma_{1} \sigma_{2} \sigma_{1} \sigma_{2} \sigma_{1} \sigma_{2} \sigma_{1} \sigma_{2}^{-q+1} \sigma_{2}^{-1} \equiv \sigma_{2} \sigma_{1} \sigma_{2} \sigma_{1} \sigma_{2} \sigma_{1} \sigma_{1} \sigma_{2}^{-q+1} \sigma_{2}^{-1} \\
& \equiv \sigma_{1} \sigma_{2} \sigma_{1} \sigma_{2} \sigma_{1} \sigma_{1} \sigma_{2}^{-q+1} \equiv \sigma_{2} \sigma_{1} \sigma_{2} \sigma_{2} \sigma_{1} \sigma_{1} \sigma_{2}^{-q+1} \\
& \equiv \sigma_{1} \sigma_{2} \sigma_{2} \sigma_{1} \sigma_{1} \sigma_{2}^{-q+2}
\end{aligned}
$$

Thus, $c(L) \leq 3+q \leq \operatorname{det}(L)$.

If $s=1, p>1$ and $q=1$, then $\operatorname{det}(L)=4+p$, and

$$
\begin{aligned}
\left(\sigma_{1} \sigma_{2}\right)^{3} \sigma_{1}^{p} \sigma_{2}^{-1} & \equiv \sigma_{1} \sigma_{2} \sigma_{1} \sigma_{2} \sigma_{1} \sigma_{2} \sigma_{1}^{p} \sigma_{2}^{-1} \equiv \sigma_{2} \sigma_{1} \sigma_{2} \sigma_{1} \sigma_{2} \sigma_{1} \sigma_{1}^{p} \sigma_{2}^{-1} \\
& \equiv \sigma_{1} \sigma_{2} \sigma_{1} \sigma_{2} \sigma_{1} \sigma_{1}^{p} \equiv \sigma_{1} \sigma_{1} \sigma_{2} \sigma_{1} \sigma_{1}^{p+1} \equiv \sigma_{1}^{p+4}
\end{aligned}
$$

Thus, $c(L)=p+4=\operatorname{det}(L)$.

If $s=1, p=1$ and $q=1$, then $\operatorname{det}(L)=5$ and

$$
\begin{aligned}
\left(\sigma_{1} \sigma_{2}\right)^{3} \sigma_{1} \sigma_{2}^{-1} & \equiv \sigma_{1} \sigma_{2} \sigma_{1} \sigma_{2} \sigma_{1} \sigma_{2} \sigma_{1} \sigma_{2}^{-1} \equiv \sigma_{2} \sigma_{1} \sigma_{2} \sigma_{1} \sigma_{2} \sigma_{1} \sigma_{1} \sigma_{2}^{-1} \\
& \equiv \sigma_{1} \sigma_{2} \sigma_{1} \sigma_{2} \sigma_{1}^{2} \equiv \sigma_{1} \sigma_{1} \sigma_{2} \sigma_{1} \sigma_{1}^{2} \equiv \sigma_{1}^{2} \sigma_{2} \sigma_{1}^{3} \equiv \sigma_{1}^{5} .
\end{aligned}
$$

Thus, $c(L)=5=\operatorname{det}(L)$.

The two other cases in Baldwin's theorem involve only a finite number of knots. A routine case by case check shows that the result holds for all these knots. This ends the proof of Conjecture 3.8 in the case of closed 3-braids.

\section{References}

[1] J A Baldwin, Heegaard Floer homology and genus one, one-boundary component open books, J. Topol. 1 (2008) 963-992 MR2461862

[2] D Bar-Natan, S Morrison, The Mathematica package KnotTheory: The knot atlas Available at http://katlas.math.toronto.edu/wiki/ 
[3] J S Birman, On the Jones polynomial of closed 3-braids, Invent. Math. 81 (1985) 287-294 MR799267

[4] R D Brandt, W B R Lickorish, K C Millett, A polynomial invariant for unoriented knots and links, Invent. Math. 84 (1986) 563-573 MR837528

[5] J C Cha, C Livingston, KnotInfo: Table of knot invariants Available at http:// www . indiana.edu/ knotinfo

[6] A Champanerkar, I Kofman, Twisting quasialternating links, Proc. Amer. Math. Soc. 137 (2009) 2451-2458 MR2495282

[7] A Champanerkar, P Ording, A note on quasialternating Montesinos links arXiv: 1205.5261

[8] J E Greene, Homologically thin, nonquasialternating links, Math. Res. Lett. 17 (2010) 39-49 MR2592726

[9] J E Greene, A spanning tree model for the Heegaard Floer homology of a branched double-cover, J. Topol. 6 (2013) 525-567 MR3065184

[10] J E Greene, L Watson, Turaev torsion, definite 4-manifolds and quasialternating knots, Bull. Lond. Math. Soc. 45 (2013) 962-972 MR3104988

[11] C F Ho, A new polynomial for knots and links: Preliminary report, Abstracts Amer. Math. Soc. 6 (1985) 300

[12] S Jablan, Tables of quasialternating knots with at most 12 crossings arXiv: 1404.4965

[13] T Kanenobu, Examples on polynomial invariants of knots and links, Math. Ann. 275 (1986) 555-572 MR859330

[14] L H Kauffman, An invariant of regular isotopy, Trans. Amer. Math. Soc. 318 (1990) 417-471 MR958895

[15] W B R Lickorish, M B Thistlethwaite, Some links with nontrivial polynomials and their crossing-numbers, Comment. Math. Helv. 63 (1988) 527-539 MR966948

[16] C Manolescu, An unoriented skein exact triangle for knot Floer homology, Math. Res. Lett. 14 (2007) 839-852 MR2350128

[17] C Manolescu, PS Ozsváth, On the Khovanov and knot Floer homologies of quasialternating links, from: "Proceedings of Gökova Geometry-Topology Conference 2007", (S Akbulut, T Önder, R J Stern, editors), GGT, Gökova (2008) 60-81 MR2509750

[18] K Murasugi, On closed 3-braids, Memoirs of the Amer. Math. Soc. 151, Amer. Math. Soc. (1974) MR0356023

[19] P S Ozsváth, J Rasmussen, Z Szabó, Odd Khovanov homology, Algebr. Geom. Topol. 13 (2013) 1465-1488 MR3071132

[20] PS Ozsváth, Z Szabó, On the Heegaard Floer homology of branched double-covers, Adv. Math. 194 (2005) 1-33 MR2141852 
[21] K Qazaqzeh, The Khovanov homology of a family of three-column pretzel links, Commun. Contemp. Math. 13 (2011) 813-825 MR2847230

[22] K Qazaqzeh, N Chbili, B Qublan, Characterization of quasialternating Montesinos links, J. Knot Theory Ramifications 24 (2015) MR3319679

[23] K Qazaqzeh, I Mansour, On Kanenobu knots arXiv: 1405.0683 to appear in Kobe J. Math.

[24] K Qazaqzeh, B Qublan, A Jaradat, A remark on the determinant of quasialternating links, J. Knot Theory Ramifications 22 (2013) MR3070840

[25] A Shumakovitch, KhoHo pari package Available at http://www.geometrie.ch/ KhoHo/

[26] M Teragaito, Quasialternating links and Q-polynomials, J. Knot Theory Ramifications 23 (2014) MR3298211

Department of Mathematics, Faculty of Science, Kuwait University

PO Box 5969, Safat-13060, Kuwait, State of Kuwait

Department of Mathematical Sciences, College of Science UAE University

15551 Al Ain, United Arab Emirates

khaled@sci.kuniv.edu.kw, nafaachbili@uaeu.ac.ae

http://faculty.uaeu.ac.ae/nafaachbili

Received: 8 July $2014 \quad$ Revised: 28 September 2014 